\title{
Nouvelles récoltes de Chironomidés (Diptera) du Maroc
}
A. Azzouzi 1
H. Laville ${ }^{2}$
F. Reiss ${ }^{3}$

Mots clés : Diptera, Chironomidae, liste faunistique, biogéographie, Maroc.

Un deuxième inventaire des Chironomidés du Maroc est donné. 87 taxa, la plupart identifiés à l'espèce, sont ajoutés à la première liste portant à 223 le total des espèces actuellement connues du Maroc.

La majorité a une large répartition paléarctique ; 7 espèces ont une distribution afrotropicale intéressante ; 10 espèces peuvent être considérées comme des endémiques de la région Sud-Ouest paléarctique.

\section{New collections of chironomids (Diptera) from Moroceo}

Keywords : Diptera, Chironomidae, faunistic list, biogeography, Morocco.

A second list of chironomids of Morocco is given. 87 taxa, most of which are identified to species, are added to the first list. The total number of species now recorded for Moroceo is 223.

The majority of species have a broad palearctic distribution ; 7 species have an interesting afrotropical distribution ; 10 species can be considered as endemic to the Southwest palearctic region.

\section{Introduction}

Le premier inventaire faunistique des Chironomidés du Maroc avait recensé 134 espèces (Azzouzi \& Laville 1987).

Une étude du réseau hydrographique de l'Oued Tensift sur le versant Nord du Haut-Atlas central, au Sud de Marrakech, a permis d'augmenter cette liste de " plusieurs " espèces nouvelles pour le territoire marocain et constitue le premier travail intensif consacré à ces diptères en Afrique du Nord (Azzouzi 1992).

A cette nouvelle liste est associée la collection des espèces marocaines encore inédites de la Zoologische Staatssammlung München (ZSM) provenant de

1. Laboratoire d'Hydrobiologie, Université Cadi Ayyad, Faculté des Sciences, Boulevard Prince My Abdellah, Marrakech, B.P. 515, Maroc.

2. Laboratoire d'Hydrobiologie, URA-695 CNRS, Université Paul Sabatier, 118, route de Narbonne, 31062 Toulouse Cédex, France.

3. Zoologische Staatssammlung, Münchhausenstrasse 21 , D-8000 München 60, Allemagne. récoltes dispersées plus particulièrement dans le Sud du Haut-Atlas (F. Reiss, ident.). Le premier inventaire a été mis à jour suivant les récentes modifications taxonomiques et les citations antérieures à 1987 omises à sa parution (Dowling 1983, 1987 ; El Mezdi \& Giudicelli 1986).

\section{Réseau hydrographique de l'Oued Tensift (Fig. 1)}

- 11 stations réparties dans 5 affluents rive gauche de l'Oued Tensift, issus du versant Nord du Haut-Atlas et échelonnées entre $1600 \mathrm{~m}$ (Oued R'Dat 1) et $550 \mathrm{~m}$ (Oued R'Dat 3), ont été échantillonnées à l'aide de filets à dérive à 4 périodes de l'année - Janvier, Avril, Juillet, Septembre 1989.

- 5 stations entre $440 \mathrm{~m}$ et $500 \mathrm{~m}$ sur le moyen Oued Tensift, rivière de plaine, réparties à l'amont (1) et à l'aval (4) de la ville de Marrakech (400 000 habitants) ont été suivies mensuellement de Janvier à Décembre 1989.

- 4 stations d'altitude situées sur l'Oued Reghaya (RG1, $3200 \mathrm{~m}$ et $\mathrm{RG} 2,1740 \mathrm{~m}$ ), sur l'Assif 
Oukaimeden (OR1, $2600 \mathrm{~m}$ ) et sur l'Assif Tiferguine (OR2, $2500 \mathrm{~m}$ ), deux affluents de l'Oued Ourika, n'ont pu être échantillonnées qu'à une seule date, en été 89.

Les principales caractéristiques abiotiques (température, $\mathrm{pH}$, conductivité, chlorures) de ces $16 \mathrm{sta}$ tions sont récapitulées sur le tableau 1.

Les stations de moyenne altitude $(1000 \mathrm{~m}$ $1600 \mathrm{~m}$ ) ont une amplitude thermique qui varie entre $9^{\circ} \mathrm{C}$ et $14^{\circ} \mathrm{C}$ (Tableau 1). Exceptionnellement, celle du N'Fis à $1600 \mathrm{~m}$ atteint $18^{\circ} \mathrm{C}$ et reflète son alimentation par des résurgences d'eau chaude.
Les amplitudes thermiques des 5 stations du Tensift, également alimentées par des rés urgences chaudes de la nappe du Haouz, varient intre $18^{\circ} \mathrm{C}$ et $20^{\circ} \mathrm{C}$; les maxima atteignent $27^{\circ}$ à $34^{\circ} \mathrm{C}$ du fait du faible couvert végétal.

Les $\mathrm{pH}$ sont toujours légèrement alcalins et varient peu en cours d'année.

Les fortes valeurs de la conductivité du R'dat 3 et du Tensift traduisent leurs fortes teneurs en chlorures dues au lessivage des terrains salifères du Permo-Trias.

Les eaux chlorées de l'Oued Zat et surtout de l'Oued R'dat débouchent dans le Tensift dont la

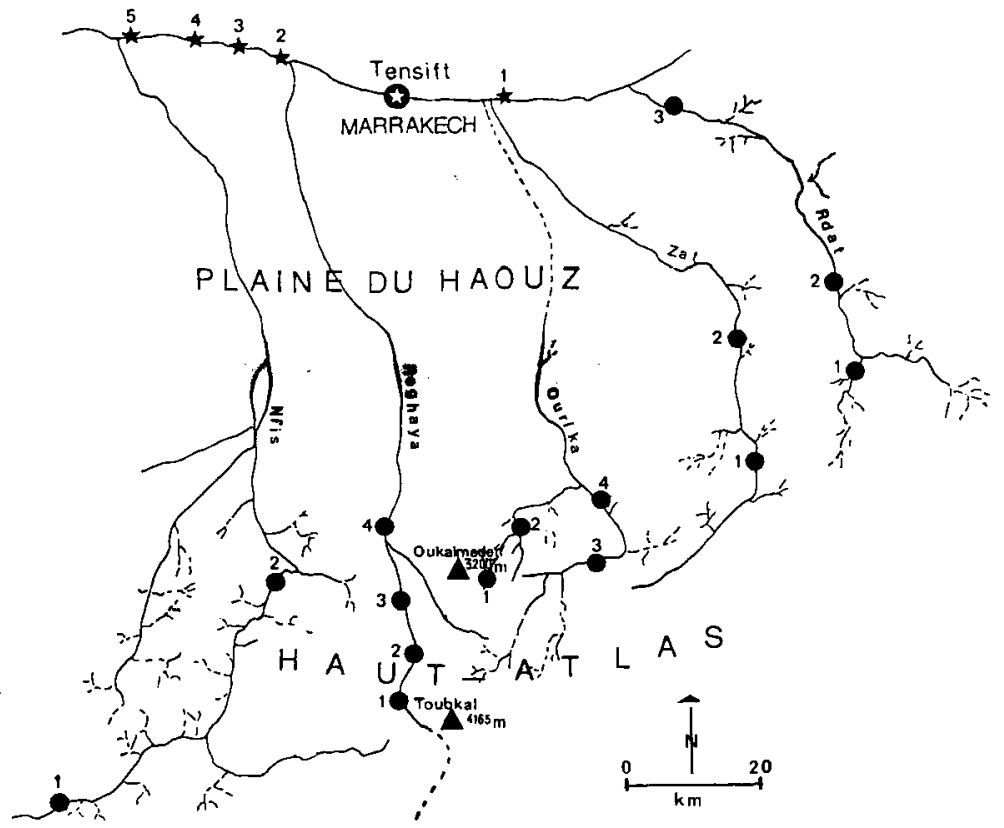

Fig. 1. Reseau hydrographique central de l'Oued Tensift : emplacement des 20 stations prospectées.

Fig. 1. The Oued Tensift, central hydrographic system : position of the 20 examined sites. 
Tableau 1. Caractéristiques et principaux facteurs abiotiques des 16 stations du réseau hydrographique de l'Oued Tensift.

Table 1. Characteristics and main abiotic factors of the 16 stations in the Oued Tensift hydrographic system.

\begin{tabular}{|c|c|c|c|c|c|c|c|c|c|c|}
\hline stations & Alt. & $\begin{array}{l}\mathrm{DS} \\
\mathrm{km}\end{array}$ & Min. & $\begin{array}{l}{ }^{\circ} \mathrm{C} \\
\operatorname{Max} .\end{array}$ & $\operatorname{Min}$. & Max. & $\begin{array}{l}\text { Cond. } \\
\text { Min. }\end{array}$ & (ps/cm) & $\begin{array}{l}\mathrm{Cl}^{-} \\
\mathrm{Min} .\end{array}$ & $\begin{array}{l}(\mathrm{mg} / 1) \\
\cdot \operatorname{Max} .\end{array}$ \\
\hline RD1 & 1600 & 15 & 9 & 20 & 7,6 & 8,3 & 175 & 430 & 18 & 142 \\
\hline $\mathrm{NF} 1$ & 1600 & 26 & 9 & 27 & 7,5 & 8,0 & 133 & 498 & 36 & 71 \\
\hline RG3 & 1550 & 68 & 8 & 17 & 7,6 & 8,3 & 150 & 360 & 18 & 70 \\
\hline OR3 & 1480 & 39 & 5 & 18 & 7,7 & 8,1 & 65 & 91 & 28 & 64 \\
\hline RD2 & 1230 & 45 & 10 & 22 & 7,6 & 8,2 & 308 & 645 & 51 & 326 \\
\hline OR4 & 1011 & 13 & 10 & 22 & 7,8 & 8,3 & 102 & 510 & 35 & 64 \\
\hline$N F 2$ & 1010 & 37 & 11 & 28 & 7,8 & 8,4 & 215 & 500 & 53 & 142 \\
\hline RG4 & 1009 & 13 & 12 & 26 & 7,8 & 8,2 & 315 & 720 & 40 & 542 \\
\hline $2 A 1$ & 1000 & 28 & 9 & 20 & 7,5 & 8,1 & 213 & 585 & 24 & 66 \\
\hline $\mathrm{ZA2}$ & 800 & 38 & 11 & 25 & 7,8 & 8,2 & 215 & 450 & 25 & 80 \\
\hline RD 3 & 550 & 105 & 11 & 28 & 7,6 & 8,0 & 830 & 3320 & 612 & 1720 \\
\hline$T F 1$ & 440 & 31 & 11 & 30 & 7,7 & 8,2 & 730 & 5860 & 987 & 3623 \\
\hline TF2 & 440 & 45 & 13 & 33 & 7,5 & 8,7 & 844 & 5110 & 787 & 3450 \\
\hline TF3 & 380 & 53 & 14 & 32 & 7,8 & 8,1 & 776 & 4754 & 1009 & 3905 \\
\hline TF4 & 335 & 61 & 15 & 34 & 7,8 & 8,2 & 1394 & 6200 & 847 & 3434 \\
\hline TF5 & 300 & 92 & 14 & 27 & 7,8 & 8,3 & 536 & 4200 & 639 & 3221 \\
\hline
\end{tabular}

salinité moyenne est de $2,5 \mathrm{~g} / \mathrm{l} \mathrm{Cl}^{-}$en période d'étiage (Juillet-Octobre) et peut atteindre jusqu'à $3,9 \mathrm{~g} / \mathrm{l}$ en Mars.

\section{Stations et dates des récoltes des Chiro- nomidés de la Zoologische Staatssammlung München : ZSM}

1. 18/08/79: Ouarzazate, $1160 \mathrm{~m}$, leg G. Fontain, capture à la lumière.

2. 02/05/79: Oasis Meski, Sud du Haut-Atlas : "Source bleue ", $25 \mathrm{~m}$ de $\mathrm{Ziz}$, $1160 \mathrm{~m}$, capture à la lumière, leg F. Auer/K. Werner.

3. 16/08/79 : Oasis Meski, leg Fontain.

4. 04/05/79 : Sud Haut-Atlas, $1140 \mathrm{~m}$, Ouarzazate, près lac de retenue, leg F. Auer.

5. 01/05/79: Moyen-Atlas, lac Aguelmane Azigza, $1510 \mathrm{~m}$, leg F. Auer' K. Werner.

6. 17/08/79: Sud Haut-Atlas, Gorges du Todra, $1400 \mathrm{~m}$, leg G. Fontain.

7. 03/05/79 : Sud Haut-Atlas, $2500 \mathrm{~m}$, entre Gorges du Todra et Gorges du Dadès, leg F. Auer/K. Werner.
8. 05/05/79 : Sud Haut-Atlas, $1540 \mathrm{~m}$, près Amergane, Vallée Asit Imini (Amont Imini), leg F. Auer/ K. Werner.

9. 06/04/80 : Ait Saoun, entre Ouarzazate et Zagora, leg K. Warncke.

10. 03/07/87 : Haut-Atlas, Gorges du Dadès, près Imdiazen, $1900 \mathrm{~m}$, leg W. Schacht.

A. Juillet 68 : Vallée du Drâa. Coll. Choumara.

B. Juillet 68 : Tissint, Moyen Drâa, Coll. Choumara $n^{\circ} 1220$.

C. Juin 68 : Environs Tetouan, Coll. Choumara.

D. Juin 68 : Marrakech, Coll. Choumara.

E. Juin 68 : Environs Larache, leg Choumara.

F. Juin 68 : Environs Berkane, leg Choumara $\mathrm{n}^{\circ} 1378$.

\section{Mise à jour du premier inventaire du Maroc}

Ces corrections concernent, le plus souvent, des changements de nomenclature, des synonymies, des descriptions de nouvelles espèces.

Les espèces précédées d'un + sont des citations omises de la littérature avant 1987, celles précédées d'un - sont des citations modifiées ou erronnées 
et sont à soustraire du premier inventaire (Azzouzi \& Laville 1987).

Paramerina Pel Langton 1984 : voir discussion Langton 1991.

Rheopelopia n.sp. Reiss : Thienemannimyia non ètudié.

+ Rheopelopia murrayi Dowling 1983 : citation omise.

- non Tanypus ? brevipalpis K. : Tanypus punctipennis (Meig.).

- Tanypus Pe1 Langton 1984 : Tanypus punctipennis (Meig.) (Langton 1991).

? Thienemannimyia n.sp. 1 Reiss: Thienemannimyia choumara $\mathrm{n} . \mathrm{sp}$. Dowling 1983.

Brillia longifurca K. syn. : Brillia flavifrons Johansen (Langton 1991).

- Cardiocladius n.sp. Reiss : Cardiocladius fuscus (Halvorsen, in litt.).

+ Corynoneura lacustris Edw. : citation omise (El Mezdi \& Giudicelli 1986).

Orthocladius Pel (Langton 1984) : Rheorthocladius sp. A. Thienemann 1944 (Langton 1991)'.

Orthocladius excavatus Br. : Orthocladius obumbratus Johannsen.

Orthocladius luteipes G. (Langton 1984) : Orthocladius rivicola $\mathrm{K}$. (Langton 1991).

Orthocladius rivicola K. (Langton 1984) : Orthoctadius ashei Soponis (Langton 1991).

Orthocladius saxicola $\mathrm{K}$. : Orthocladius rubicundus (Meigen).

+ Dicrotendipes peringueyanus (K.) : citation ornise (Ramdani \& Tourenq 1982).

Dicrotendipes pilosimanus K. syn. : Dicrotendipes septemmaculatus (Becker) (Cranston \& Armitage 1988).

- Dicrotendipes collarti G. : citation omise (El Mezdi \& Giudicelli 1986).

- Paratendipes striatus (K.) : citation omise (El Mezdi \& Giudicelli 1986).

- Pentapedilum ? n.sp. (Marokko) Reiss : Polypedilum nubens (Edwards).

+ Polypedilum ruandae Freeman : citation omise (El Mezdi \& Giudicelli 1986).

+ Polypedilum tridens Freeman : citation omise (EI Mezdi \& Giudicelli 1986).

Stictochironomus n.sp. : Stictochironomus reissi Cranston 1989.

Cladotanytarsus Pe5 (Langton 1984) : Cladotanytarsus pallidus Kieffer (Langton 1991).

+ Cladotanytarsus capensis (Freeman) : citation omise (El Mezdi \& Giudicelli 1986).

- Tanytarsus ? brevipalpis G. : citation erronée ; à supprimer.

1. O. ruffoi Rossaro (Rossaro 1992).
Tanytarsus n.sp. (creticus) Reiss : Tanytarsus cretensis Reiss 1987.

Tanytarsus n.sp. (Marokko) Reiss : Cladotanytarsus ecrisfatus Reiss 1991.

Tanytarsus Pe5 Langton 1984 : Tanytarsus signatus van der Wulp (Langton 1991).

Virgatanytarsus maroccanus Kugl. \& Reiss syn. : Virgatanytarsus albisutus (Santos Abreu) (Cranston \& Armitage 1988).

Dans le premier inventaire, 134 espèces ou taxa éraient dénombrés. Après cette mise à jour, 5 espèces $(-)$ disparaissent et 8 espèces $(+)$ s'ajoutent, portant à 137 le total des espèces réellement recencées du Maroc jusqu'en 1987. Le tableau 2 récapitule la liste des 86 espèces ou taxa de chironomidés cités pour la première fois du Maroc après 1987, avec leurs stations de récoltes.

\section{Discussion}

La majorité des nouvelles citations de la ZSM concernent des Chironominae, celles du réseau hydrographique du Tensift se rapportent principalement aux Orthocladiinae.

Ce nouvel inventaire porte à 223 le total des espèces de Chironomidés actuellement recensés du Maroc.

Les espèces se répartissent dans les principales sous-familles et tribus selon le tableau 3 .

L'actuel inventaire des Chironomidés du Maroc est loin d'être exhaustif. Sur la base des 598 espèces recensées en France continentale et de Corse (SerraTosio \& Laville 1991), il représente probablement le tiers des espèces potentielles de l'ensemble du territoire marocain dont la grande diversité des milieux en zone aride, semi-aride et humide n'a pas été prospectée de façon intensive.

Les récoltes actuelles concernent une sélection d'habitats essentiellement lotiques et, plus accessoirement lentiques, de plaine et de moyenne montagne.

Les éléments terrestres, les krénobiontes, particulièrement des sources froides de haute montagne, les halobiontes restent largement sous-représentés dans cet inventaire.

La majorité $(86,5 \%)$ de la faune des Chironomidés actuellement recensés du Maroc est d'origine paléarctique comme c'est le cas chez beaucoup d'autres groupes d'insectes.

D'un point de vue écologique et biogéographique, les Khettaras du Sud de Marrakech apparaissent 
Tableau 2. Inventaire et stations de récoltes des Chironomidés nouveaux pour le Maroc.

+ : stations du réseau hydrographique de l'Oued Tensift. Chiffres et lettres : références des stations ZSM.

* : Exuvies nymphales nouvelles.

Table 2. List and sampling sites for chironomids new to Morocco.

+ : sites of the Oued Tensift hydrograptic system. Numbers and letters : references of the ZSM sites.

$\because$ new pupal exuviae.

Tanypodinae (16)

Ablabesmyia langistyla rittkau Conchapelopia viator (Kieffer)

Larsia $?$ curticalcar (Kieffer) Larsia atrocincta (Goetghebuer) Macropelopia nebulosa (Meigen) Nilotanypus dubius (Meigen) paramerina cingulata (Walker\} Procladius brevipetiolatus Goetghebuer procladius noctivagus (Kieffer)

Tanypus kratzi (Kieffer)

Thienemannimyia berkaned Dowling 1987

Thienemannimyia ? laeta (Meigen)

Thienemannimyia lentiginosa (Fries)

Trissopelopis longimana (staeger)

zavrelinyia melanura (Meigen\}

- Pentaneurelia sp ourika

Podominae (1)

Paraboreochlus ninutissimus (strobl)

Diamesinae (4)

Boreoheptagyini

Boreoheptagyia legeri (Goetghebuer)

Diamegini

Dienesa vaillanti Serra-Tosio

Dianesa ? zernyi Edwards

sympothastie zavreli pagast

Prodianesinae

(1)

prodiamesa olivacea (Meigen)

Orthocladiinae (38)

Brillia modesta (Meigen)

Chaetocladius gr. acuticornis

Chetocladius gr. vitellinus

Corynoneura Iobata Edwards

corynonenra Pe2b Langton 1991

Cricotopus annulator Gotghebuer

cricotopus beckeri Hirvenoja

Cricotopus brevipalpis Kieffer

cricotopus ornatus (Meigen)

Cricotopus pallidipes Edwards

Eukiefferiella brevicalcar (Rieffer)

Eukiefferiella fittkaui Lehmann

Eukiefferiella gracei (Edwards)

Eukiefferiella tiralensis Goetghebuer

Krenosmittia camptophleps (Edwards)

Metriocnemus obscuripers (Holngren)
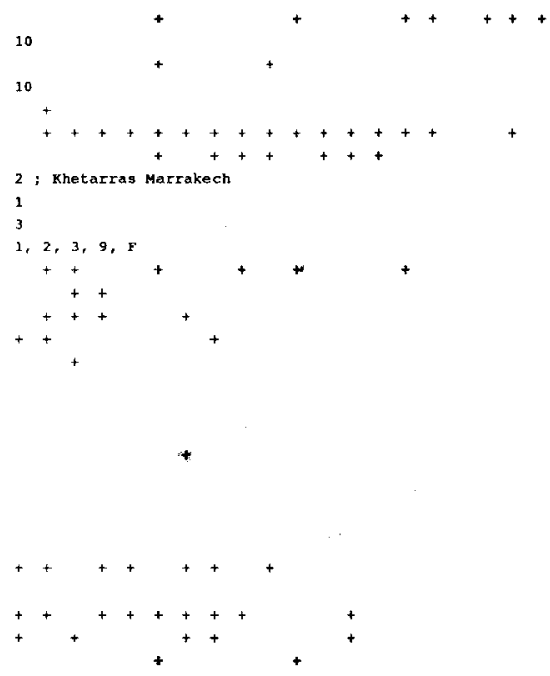

$+$

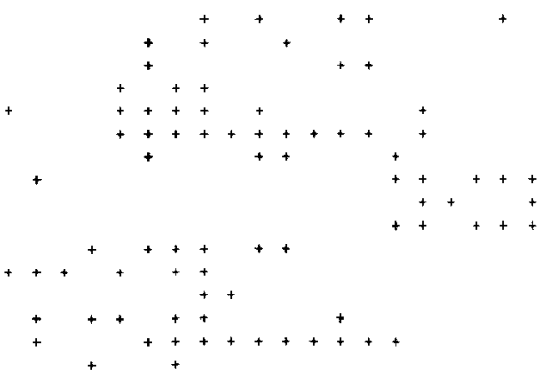


orthocladi inae gen. 7 sp. 7 Pel Langton 1991 (cf. Rrenosmittia hispanica Wulker) orthocladius fuscimanus Kieffer orthocladiug ablidens Goetghebuer orthocladius rivulorum Kieffer Orthocladius thienemanni Kieffer Parakiefferiella coronata (Edwards) Parakiefferiel la sp. d wilker

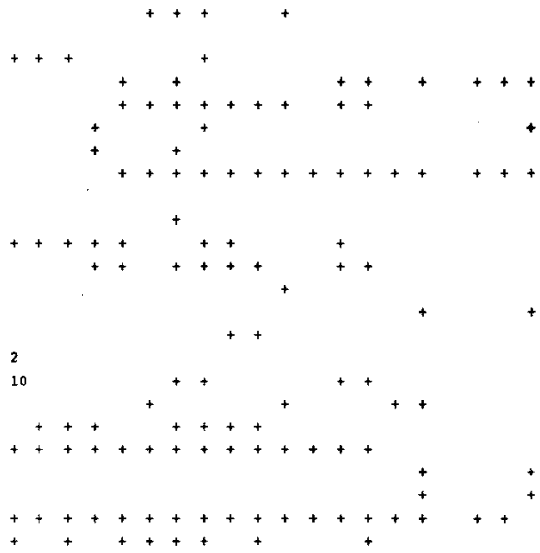

Parametrioanemus sp. Rheghaya

Paratrichocladiug skirwithensis (Edwards) Parorthocladius nudipennis (Kieffer)

Psectrocladius obvius (walker)

Psectrocladius ventricosus kieffer Pseudorthocladius berthelenyi Moubayed Pseudorthocladius curtistylus (Goetghebuer) Rheocricotopus atripes (Kieffer) Rheocricotopus fuscipes (Kieffer) Rheocicotopus tirolus Lehmann synorthocladius semivirens (Kieffer) Trissocladius brevipalpis kieffer

- Trissocladius sp. Tensift

Tretenia calvescens (Edwards)

Tvetenia veralli (Edwards)

chironombare (26)

Chironomini (13)

Chironomini gen. $\hat{\text { n.sp. }}$ ? Pe\$ Langton 1991 Cryptotendipes graminicolor (Rieffer) Dicrotendipes fusconotatus (Rleffer) Harnischia 7 n.sp. Reiss

Kloosia pusilla (Linnaeus)

Microchironomus tener (Kieffer)

Microtendipes confinis (Meigen)

Paxacladopelma ? galaptera (Townes)

Paraciadopelma mikiana (Goetghebuer)

palypedilum arundineti (Goetghebuer)

Polypedilum latum (Meigen)

polypedilur tetracrenat um Birvenoja

Polypedilum Pel Langton 1991

Tanytarsini (13)

Cladotanytarsus ecristatus Reias 1991

Lithotanytarsus dadesi Reing 1991

Micropsectra bidentata Goetghebuer

micropsectra contracta Reiss

Hicropsectra junci (Meigen)

Micropsectre I indrothi Goetghebuer

Paratanytarsus hituberculatus (Edwards)

Paratanytarsus dissimilis Johannen

Rheotanytarsus curtistylug (Goetghebuer)

Rheotanytarsus procerus Reiss 1991

Tanytarsus chinyensis Goetqhebuer

Virgatanytarsus arduensensis (Goetghebuer)

virgatanytarsus triangularis (Goetghebuer)

$9,10, \mathrm{~A}, \mathrm{D}$

10

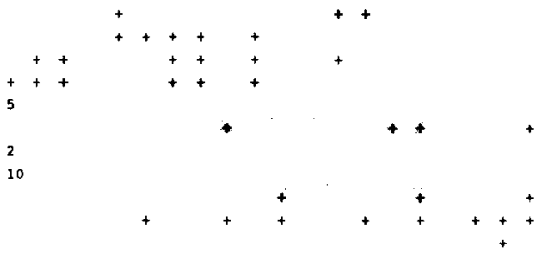


Tableau 3. Répartition des Chironomidés recensés au Maroc avant (A) et après (B) 1987.

Table 3. Distribution of chironomids recorded from Morocco before (A) and after (B) 1987.

\begin{tabular}{lrrrc}
\hline & A & B & Total & $\%$ \\
\hline Tanypodinae & 15 & 16 & 31 & 13,9 \\
Podonominae & & 1 & 1 & \\
Diamesinae & 8 & 4 & 12 & 5,4 \\
Prodiamesinae & & 1 & 1 & \\
Orthocladiinae & 42 & 38 & 80 & 35,9 \\
Chironominae & & & & \\
Chironomini & 49 & 13 & 62 & 27,8 \\
Tanytarsini & 23 & 13 & 36 & 16,1 \\
Total & 137 & 86 & 223 & \\
\hline
\end{tabular}

comme des milieux particulièrement intéressants puisqu'ils abritent une faune d'au moins 4 espèces d'origine Afrotropicale (El Mezdi \& Giudicelli 1986) :

Dicrotendipes collarti: S-Maroc, Zaïre, Kenya, Malawi.

Paratendipes striatus : S-Maroc, Tchad, Soudan, Egypte.

Polypedilum ruandae: S-Maroc, Zaïre, Afrique du Sud.

Polypedilum tridens : S-Maroc, Zaire, large répartion Afrotropicale (Madagascar incl.), Egypte.

Cladotanytarsus capensis, également citée de ces Khettaras, est une forme très semblable à Cladotanytarsus pseudomancus $\mathrm{G}$. dont elle n'est peut-être qu'une variété sombre (Freeman 1958).

Ces Khettaras sont un système d'irrigation traditionnel des régions arides ou semi-arides où l'eau de la nappe phréatique est conduite à la surface.

Les conditions thermiques $\left(19^{\circ}-23^{\circ} \mathrm{C}\right)$ et hydrologiques stables ont permis l'installation d'une faune invertébrée originale.

Ces 4 espèces d'origine afrotropicale n'ont jamais été trouvées dans le proche système du Tensift, pourtant prospecté intensivement. Ces espèces, apparemment relictes dans le Sud du Maroc suggèrent une progression intéressante par l'oueșt vẹrs la région méditerranéenne (Laville \& Reiss, sous presse).
Deux autres espèces, également afrotropicales, ont une plus large répartition dans la région méditerranéenne :

Dicrotendipes peringueyanus : Maroc, Algérie, SFrance, Espagne, Italie, Arabie Saoudite, Sénégal, Tchad, Cameroun, Zaïre, Ouganda, Zambie (= Rhodésie).

Microtendipes lendli

: S-Maroc, Turquie, Palestine, (syn. stilifer Freeman 1954), Israël, Liban, Egypte, Arabie Saoudite, Soudan, Ouganda, Afrique du Sud.

Une autre particularité du peuplement marocain concerne 10 especes que l'on peut présentement considérer comme des éléments endémiques de la région Sud-Ouest paléarctique qui peut inclure le Sud de la Péninsule Ibérique.

Paramerina maurefanica : Maroc, Mauritanie.

Rheopelopia murrayi: Maroc, Algérie.

Thienemannimyia choumara: Maroc.

Chironomini gen.? sp. ? Pe3 Langton 1991 : Maroc, Tunisie.

Harnischia n.sp. : Maroc, Espagne, Portugal.

Rheomus alatus : Maroc.

Rheomus yahiae: Maroc.

Cladotanytarsus ecristatus : S-Maroc.

Lithotanytarsus dadesi: S-Maroc.

Rheotanytarsus procerus : S-Maroc.

3 exuvies nouvelles, trouvées dans le réseau hydrographique du Tensift, seront décrites ultérieurement et peuvent momentanément être envisagées comme endémiques : Pentaneurella sp. Ourika, Parametriocnemus sp. Rheghaya, Trissocladius sp. Tensift.

Il existe également 10 espèces marocaines à répartition circum-méditerranéenne sans qu'on puisse différencier des éléments exclusivement Ouestméditerranéens (Laville \& Reiss 1993) :

Thienemannimyia berkanea : Maroc, Israël. Zavrelimyia berberi : Maroc, Liban.

Cricotopus beckeri : Maroc, S-Espagne, Madère, S-E France, Algérie, Grèce, Turquie. 
Eukiefferiella bedmari : Maroc, S-Espagne, Grèce, Turquie, Liban.

Orthocladiinae gen. ? sp. ? Pel Langton 1991 : Maroc, Tunisie, Italie, Turquie.

Pseudorthocladius berthelemyi : Maroc, S-France, Bulgarie, Turquie.

Baeotendipes noctivaga: Maroc, Tunisie, Espagne, Italie, Grèce, Bulgarie, Roumanie, URSS (SET).

Stictochironomus reissi : Maroc, Algérie, Turquie, Syrie, Iraq, Afghanistan.

Paratanytarsus mediterraneus: Maroc, Algérie, Italie, Roumanie, Turquie, Syrie.

Virgatanytarsus albisutus: Maroc, Canaries, Algérie, Grèce.

Ces considérations biogéographiques restent absolument spéculatives car on ne dispose souvent que de rares indices susceptibles d'être modifiès à la première prospection. Ce fut le cas notamment de 2 espèces nouvelles, décrites du Maroc, Thienemannimyia berkanea et Zavrelimyia berberi et signalées par la suite beaucoup plus tard à l'est, la premiàre en Israël1, la seconde au Liban (Moubayed \& Laville 1983). Il s'agit bien d'un état instantané de biorépartition, susceptible d'évoluer en fonction de recherches plus intensives dans toute la région circum-méditerranéenne.

1. Thienemannimyia berkanea : Israël, Jordan, Taufstelle des Heiligen Johannes, 30.10.1954, 1 o Imago, leg E. J. Fittkau.

\section{Travaux cités}

Azzouzi A. 1992. - Etude écologique des Chironomidés (Diptera) du réseau hydrographique central du versant Nord du Haut-Atlas (Maroc). Thèse $3^{c}$ cycle, Hydrobiologie, Univ. Cadi Ayyad, Marrakech (Maroc) : p.

Azzouzi A. \& Laville H. 1987. - Premier inventaire faunistique des Chironomidés (Diptera, Chironomidae) du Maroc. Annis Limnol., 23 (3) : 217-224.

Cranston P.S. \& Armitage P.D. 1988. - The Canary Islands Chironomidae described by T. Becker and by Santos Abreu. Dtsch. ent. Z., N.F. $35(4-5): 341-354$.

Dowling C. 1983. - A description of two new species of Tanypodinae (Diptera : Chironomidae) from North Africa. $\mathrm{Mem}$. Amer. Ent. Soc., 34 : 89-94.

Dowling C. 1987. - A description of two new species of the genus Thienemannimyia (Diptera : Chironomidae), from North Africa. Ent, Scand., Suppl. $29: 157-160$.

El Mezdi Z. \& Giudicelli J. 1986. - Etude d'un écosystème lim nique peu connu : les khettaras de la région de Marrakec (Maroc). Habitats et peuplements. Sciences de l'eau, 6 (3) 281-297.

Freeman P. 1958. - A study of the Chironomidae (Diptera) of Africa South of the Sahara. IV. Bull. Br. Mus. not. Hist. Ent., $6: 261-363$.

Langton P.H. 1984. - A key to pupal exuviae of british Chironomidae. Cambrideshire : $324 \mathrm{p}$.

Langton P.H. 1991. - A Key to pupal exuviac of West Paleartic Chironomidae. Cambridgeshire : 386 p.

Laville H. \& Reiss F. 1992. - The Chironomid fauna of the mediterranean region reviewed. Netherlands Journal of Aquatic Ecology, 26 (2-4) : (sous presse).

Moubayed Z. \& Laville H. 1983. - Les Chironomidés (Diptera) du Liban. I. Premier inventaire faunistique. Annls Limnol. 19 (3) : 219.228 .

Ramdani M. \& Toureng J.N. 1982. - Contribution à l'étude faunistique de la Merja de Sidi Bou Ghaba. Chironomidés (Diptères). Bull. Inst. Scient. Rabat, $n^{\circ} 6$ : 179-185.

Reiss F. 1987. - Tanytarsus cretensis sp.n., eine neue westpalaearktische Chironomidenart aus Fliessgewässern (Diptera. Insecta). NachrBl. bayer. Ent., $36: 26-30$.

Reiss D. 1991. - Drei neue Tanytarsini-Arten aus Marokko (Dip tera, Chironomidae). NachrBI. bayer. Ent., 40 (2) : 45-52

Rossaro B. 1992. - Chironomidae from Italy. Chironomus Newsletter, $4: 19$. 